\title{
Atomic Imaging and Spectroscopy of Two-Dimensional Materials
}

\author{
Juan Carlos Idrobo ${ }^{1}$, Wu Zhou ${ }^{2}$, Myron Kapetanakis ${ }^{3,2}$, Micah P. Prange ${ }^{4}$, Leonardo Basile ${ }^{5,1}$, Sokrates \\ T. Pantelides ${ }^{3,2}$ and Stephen J. Pennycook ${ }^{6}$ \\ ${ }^{1}$ Center for Nanophase Materials Sciences, Oak Ridge National Laboratory, Oak Ridge, TN 37831 , \\ USA \\ ${ }^{2}$ Materials Science and Technology Division, Oak Ridge National Laboratory, Oak Ridge, TN 3783,1 \\ USA \\ ${ }^{3}$ Department of Physics and Astronomy, Vanderbilt University, Nashville TN 37235, USA \\ ${ }^{4}$ Fundamental and Computational Sciences Directorate, Pacific Northwest National Laboratory, \\ Richland, WA 99352, USA \\ ${ }^{5}$ Departamento de Física, Escuela Politécnica Nacional, Quito, Ecuador \\ ${ }^{6}$ Department of Materials Science and Engineering, University of Tennessee, Knoxville, TN 37996, \\ USA
}

The introduction of aberration-correction in scanning transmission electron microscopy (STEM) has allowed the realization of Feynman's long sought dream [1], atom-by-atom structural and elemental identification of materials [2], by simply looking "at the thing". Moreover, the higher current in the electron probes achieved with aberration correction has also allowed the identification, by spectroscopic means, of individual impurities [3], as well as the bonding characteristic of individual atoms in two-dimensional (2D) materials [4-6].

In this talk, we will present our ongoing efforts to study 2D materials using STEM analytical techniques combined with first-principles total energy calculations. We will also discuss the new doors that will be opened by advances in instrumentation in analytical STEM. For instance, electron probes with even better spatial and energy resolution of than is currently available ( $<0.5 \mathrm{~A}$ and $<10 \mathrm{meV}$, respectively), as well as the combination with in-situ measurements, such as heating and biasing.

Figure 1 shows an example of single atom spectroscopy performed in Oak Ridge National Laboratory's aberration-corrected Nion UltraSTEM ${ }^{\mathrm{TM}} 100$, which has a cold field-emission electron source and that can correct $3^{\text {rd }}$ and $5^{\text {th }}$-order aberrations [7]. The left panel in Fig. 1 shows two Z-contrast images of individual Si impurities in graphene adopting three- and four-fold coordination. However, the images do not reveal if the Si atoms are bonded in or out of plane of the carbon atoms. Atomically resolved EEL spectra (Fig.1 right panel) acquired for the Si impurities reveal different features in their Si L-edges (i.e., the peak at $105 \mathrm{eV}$ ), indicating different mixing of the Si $3 d$ states with the surrounding carbon atoms. First-principles calculations explain the EEL result by showing that for the case of the three-fold coordinated $\mathrm{Si}$ atom, the $\mathrm{Si} 3 d$ orbitals contribute significantly to the bonding, resulting in a planar $\mathrm{sp}^{2} \mathrm{~d}$-like hybridization. In contrast, the three-fold coordinated $\mathrm{Si}$ in graphene adopts the preferred $s p^{3}$ hybridization for $\mathrm{Si}$, with the $\mathrm{Si}$ atom out of plane by $0.05 \mathrm{~nm}$ [7].

\section{References:}

[1] R.P. Feynman, "There is plenty of room at the bottom”, (1959).

[2] O.L. Krivanek et al., Nature 464, (2010), 571.

[3] M. Varela et al. Phys. Rev. Lett. 92, (2004), 095502.

[4] K. Suenaga and M. Koshino, Nature 468, (2010), 1088. 
[5] W. Zhou et al., Phys. Rev. Lett. 109, (2012), 206803.

[6] Q. Ramasse et al., Nano Letters 13, (2013), 4989.

[7] O.L. Krivanek, et al. Ultramicroscopy 108, (2008) 179.

[8] This research was supported by the Center for Nanophase Materials Sciences (CNMS), which is sponsored at ORNL by the Scientific User Facilities Division, Office of Basic Energy Sciences, U.S. DOE (JCI), by a Wigner Fellowship through the Laboratory Directed Research and Development Program of Oak Ridge National Laboratory (ORNL), managed by UT-Battelle, LLC, for the U.S. DOE (WZ), the National Secretariat of Higher Education, Science, Technology and Innovation of Ecuador (SENESCYT) (LB), and by the Office of Basic Energy Sciences, Materials Sciences and Engineering Division, U.S. DOE (STP, SJP), and DOE Grant No. DE-FG02-09ER46554 (MDK, MPP, STP). This research used resources of the National Energy Research Scientific Computing Center, which is supported by the Office of Science of the U.S. Department of Energy under Contract No.

DE-AC02-05CH11231.
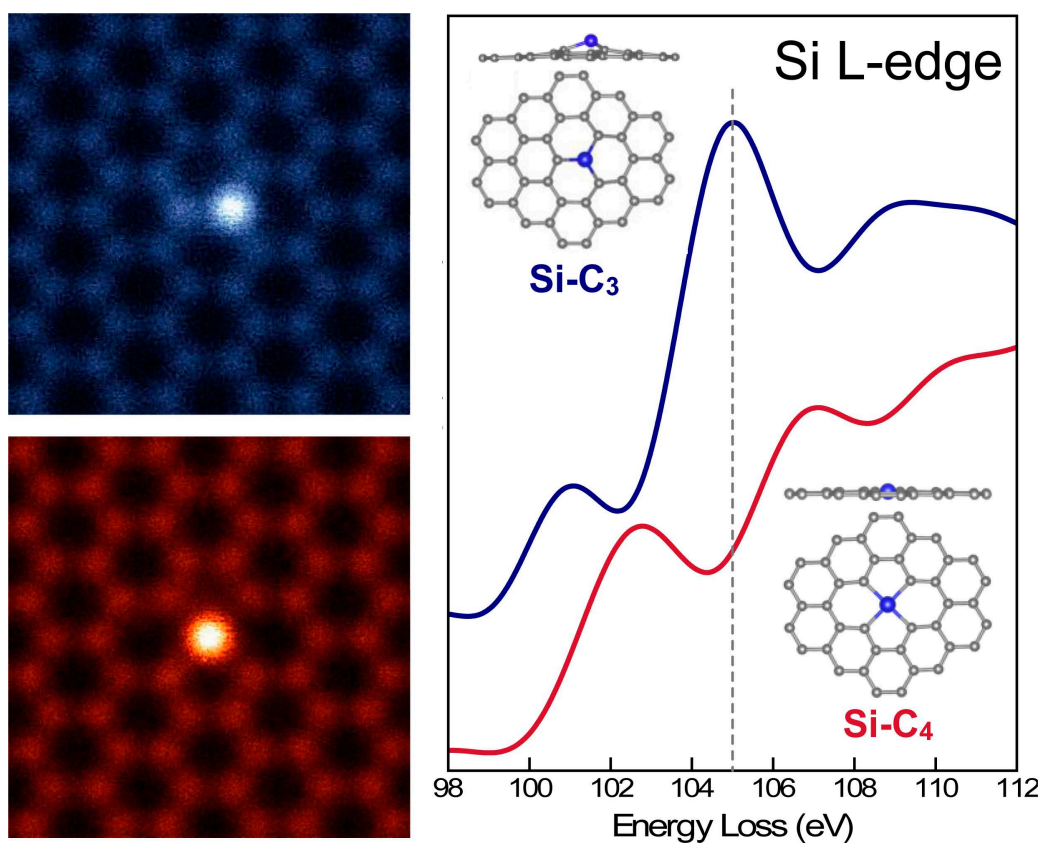

Figure 1. The atomic and electronic structure of individual Si impurities bonded to three or four carbon atoms in graphene are revealed by Z-contrast imaging (left panel), electron energy-loss spectroscopy (right panel) using an aberration-corrected scanning transmission electron microscope in combination with density functional theory calculations. Inset on the right panel; shows the atomic structure formed by the $\mathrm{Si}$ impurities obtained by first-principles calculations. Images adapted from Ref. [5]. 